\title{
Enfermedad de Buerger o tromboangeítis obliterante: importancia de un diagnóstico precoz y tratamiento con análogos de la prostaglandina E1 (PGE1)
}

\author{
Buerger's disease or obliterant thromboangeitis: Importance of accurate \\ diagnosis andtreatment with analogs of prostaglandine E1
}

Martín Najenson ${ }^{1}$, Sergio Lupo ${ }^{2}$, Claudio Cigalini' ${ }^{1}$, Carlos Dumont ${ }^{1}$, Roberto Favaloro ${ }^{3}$, Mirna Biglione ${ }^{4}$

\section{RESUMEN}

La enfermedad de Buerger (EB) es una afección inflamatoria segmentaria no aterosclerótica de los vasos sanguíneos de pequeño y mediano calibre, típica de las extremidades. De difícil diagnóstico, representa un reto debido a la alta tasa de amputaciones. Se asocia al tabaquismo y se presenta más frecuentemente en varones jóvenes. Su único tratamiento probado y aceptado es el cese del hábito tabáquico, aunque existe evidencia de que prostanoides endovenosos serían seguros y eficaces en términos de analgesia y reducción de la tasa de amputaciones en estos pacientes. Se describe un caso diagnosticado en Rosario, Argentina.

Palabras claves: enfermedad de Buerger, tromboangeítis obliterante, tabaco, isquemia crítica de miembros, síndrome del dedo azul, urgencia, prostaglandina E1.

\begin{abstract}
Buerger Disease is a non-atherosclerotic, segmental inflammatory affection of the small and medium-sized blood vessels, typical of the extremities.Difficult to diagnose,it represents a challenge due to the high rate of amputations. It is related to tabaquism and occurs more frequently in young men. The only proven and universally accepted treatment is smoking cesation, but there is evidence that intravenous prostanoids are safe and effective in terms of analgesya and in reducing the rate of amputations in these patients. We describe a case diagnosed in Rosario, Argentina.
\end{abstract}

Keywords: Buerger's disease, thromboangiitis obliterans, tobacco, critical limb ischaemia, blue finger syndrome, urgency, prostaglandin ET.

Revista Argentina de Cardioangiología Intervencionista 2019;10(3):117-119. https://doi.org/10.30567/RACl/201903/0117-0119

\section{INTRODUCCIÓN}

La EB de distribución mundial, con mayor prevalencia en países mediterráneos y asiáticos, es una afección inflamatoria segmentaria no aterosclerótica de vasos de pequeño/mediano calibre, típica de las extremidades. Se caracteriza por endarteritis que genera un estado protrombótico y subsecuentes fenómenos oclusivos ${ }^{1}$. Está asociada al consumo de tabaco; y se cree que obedecería a un mecanismo autoinmune; existiendo evidencia de hipersensibilidad a la inyección intradérmica de extractos de tabaco ${ }^{2}$.

\section{CASO CLÍNICO}

Paciente de 47 años, tabaquista (30 paquetes/año) dislipémico que consulta por cuadro de ocho meses de evolución de dolor digital de miembros inferiores (MI) de carácter urente y recurrente; de aparición espontánea o gatillado por maniobras de compresión, asociado a cambios transitorios en la coloración de los dedos (Figura 1A) y en ocasiones de dolor lumbar. En traumatología se descarta compresión radicular por RMI. A los 6 meses consulta a dermatología por cianosis focalizada en 4 to dedo derecho y 5 to izquierdo (Figura 1B); se realiza tratamiento empírico para der-

1. Hospital Privado, Rosario, Argentina

2. School of Medical Sciences (UNR) and UAI Rosario, Centralized Institute of Integral Clinical Research (CAICI), Rosario, Argentina

3. Hospital Universitario Fundación Favaloro, Buenos Aires, Argentina

4. UBA-CONICET.Instituto de Investigaciones Biomédicas en Retrovirus y SIDA, Buenos Aires, Argentina

$\triangle$ Correspondencia: Dra. Mirna Biglione, INBIRS UBA-CONICET, Paraguay 2155, C1121ABG CABA, Rep. Argentina. Tel.: (+54911)61236551.mbiglione@fmed.uba.ar Los autores no declaran conflictos de intereses

Recibido: 18/01/2019| Aceptado: 17/07/2019 matitis de contacto, sin respuesta al mismo. El dolor se torna continuo,refractario a los AINE vía oral, compromete el reposo nocturno y cede levemente con la deambulación. Agrega además lesiones digitales ulceronecrosantes (Figura 1C). Por sospecha de vasculitis, se solicita laboratorio, biopsia y se indica AAS, prednisolona, tramadol y paracetamol. Ante la consulta a cardiología, se indica suprimir totalmente el tabaquismo, amitriptilina y prasugrel, sin respuesta favorable. Se decide internación, se asocia cilostazol $(100 \mathrm{mg} / 12 \mathrm{~h})$, pentoxifilina $(400 \mathrm{mg} / 12 \mathrm{hs})$, pregabalina $(150 \mathrm{mg} / 12 \mathrm{hs})$, enoxaparina $80 \mathrm{mg} /$ día y morfina endovenosa. Como antecedentes registra hipotiroidismo, exposición a pesticidas, trauma vibratorio por motosierra, y medicación con levotiroxina y fenofibrato/ezetimibe.

El examen físico reveló pulsos normales y simétricos con índice tobillo/brazo de 1 . El test de Buerger resultó positivo a la sobreelevación de 60 grados con hiperemia reactiva durante la fase de declive. Presentaba trombocitosis $(447.000 / \mu l)$. La serología para $\mathrm{VIH}$, hepatitis, panel inmunológico (anticuerpos antinucleares, anticentrómero, C3, C4, ANCA-C y ANCA-P, scl-70, factor reumatoideo, anti-RNP) y hematológico (KPTT, crioglobulinas, anticardiolipinas, anticoagulante lúpico, inmunofijación en suero, antitrombina, factor $\mathrm{V}$ de Leiden, proteína s y c funcional, homocisteína, resistencia a proteína c, estudio de Fabry) fueron negativos. La biopsia del 4 to dedo describió vasos dilatados, telangiectasia y trombos de fibrina. Se detectó la variante p.V617F en JAK2 (heterocigota) sin criterio para trombocitosis esencial. Elecocardiograma, eco-Doppler arterial/venoso de miembros/cuello, fueron normales. La angio-TAC con contraste de tórax-abdomen-pelvis mostró esplenomegalia leve, descartándose arterosclerosis aórtica La angiografía periférica bilateral no evidenció lesiones obstructivas en todo el lecho vascular hasta los arcos plantares. Se confirmó, por exclusión y por criterios de Olin 6 , enfermedad de Buerger (EB). Ante el 


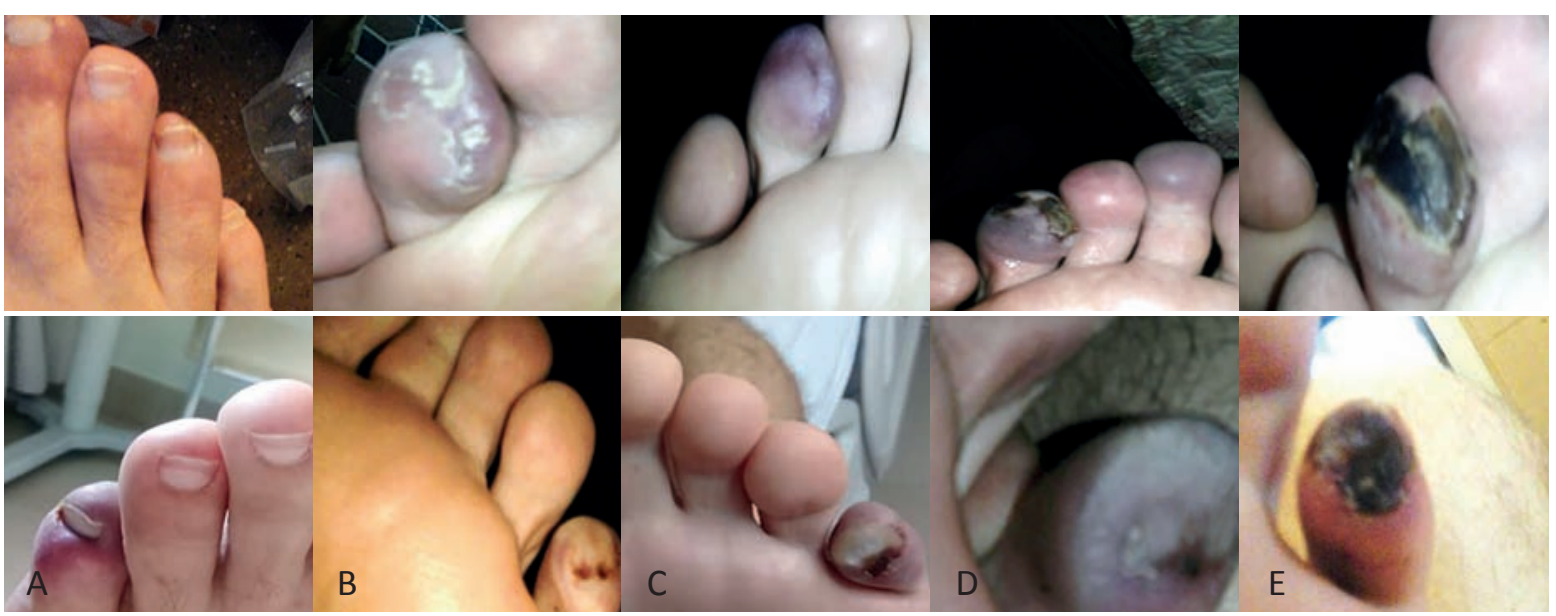

Figura 1. Evolución de lesiones isquémicas en enfermedad de Buerger previo ( $A$ a D) y a la consulta (E). Nótese evolución desde eritema (A) a cianosis ( $C$ ) y gangrena focalizada (E)

TABLA 1. Criterios diagnósticos estrictos de la enfermedad de Buerger (Oregón) y diagnóstico diferencial.

\begin{tabular}{|l|}
\hline Criterios mayores \\
\hline Inicio de síntomas isquémicos en la extremidad antes de los 50 años \\
\hline Abuso de tabaco \\
\hline Exclusión de: \\
\hline Fuente embolígena proximal \\
\hline Traumatismo local \\
\hline Enfermedad autoinmune \\
\hline Estado de hipercoagulabilidad \\
\hline Arteriosclerosis: \\
\hline Diabetes \\
\hline Hiperlipidemia \\
\hline Hipertensión \\
\hline Insuficiencia renal \\
\hline Ausencia de afectación proximal a la arteria poplítea o braquial distal \\
\hline Documentación objetiva de enfermedad oclusiva distal mediante: \\
\hline Doppler arterial segmentario y pletismografía de 4 miembros \\
\hline Arteriografía \\
\hline Histopatología (no patognomónico) \\
\hline Criterios menores \\
\hline Tromboflebitis migratoria \\
\hline Síndrome de Raynaud \\
\hline Afectación de miembros superiores \\
\hline Claudicación de dorso del pie \\
\hline Diagnóstico diferencial de Enfermedad de Buerger \\
\hline Vasculitis (sistémicas y crioglobulinémica). \\
\hline Colagenopatías (LES, artritis reumatoidea, esclerodermia, síndrome de Sjögren). \\
\hline $\begin{array}{l}\text { Ateroembolia o embolia de origen cardíaco (endocarditis bacteriana subagu- } \\
\text { da, mixoma auricular, endocarditis marántica, endocarditis de Libman-Sacks). }\end{array}$ \\
\hline Trastornos de hipercoagulabilidad adquiridos (síndrome de Trusseau, síndrome \\
anticardiolipina, necrosis por warfarina,trombocitopenia inducida por heparina). \\
\hline Enfermedad por inmunocomplejos. \\
\hline Eritema pernio. \\
\hline Gangrena periférica simétrica. \\
\hline Obteniday adaptada de Goiriz-Valdés Ry J Fernández-Herrera J' \\
\hline
\end{tabular}

progreso de las lesiones (Figura 1D), el aumento del dolor y la falta de disponibilidad de iloprost en su formulación endovenosa, se inicia tratamiento con alprostadil endovenoso (Tabla 2). Se obtuvo excelente respuesta terapéutica con mejoría del dolor que permitió el reposo nocturno a partir del segundo día y reducir la dosis de analgésicos hasta su suspensión. A los 7 días continúa tratamiento domiciliario; con cuidados locales (antibióticos, crema con colagenasa y desbridamiento) y especialista en terapia neural agrega inyecciones subcutáneas semanales de procaína $1 \%$ en raíz de dedos ${ }^{12}$. A los 15 días de tratamiento, inicia ejercicio físico (bicicleta y caminatas $20 \mathrm{~min} /$ día); continúa con medidas higiénico-dietéticas, abstinencia tabáquica, de exposición al frío, uso de calzado adecuado y soporte psicológi-
TABLA 2. Esquema de tratamiento con alprostadil a pasar durante tres horas por bomba de infusión y bajo monitoreo continuo.

\begin{tabular}{|l|c|c|c|c|c|c|}
\hline Día & 1 & 2 & $3-4$ & $5-8$ & $9-20$ & $21-28$ \\
\hline Dosis mcg/día & 40 & 60 & 80 & 100 & 120 & reducción de a 20 \\
\hline
\end{tabular}

co. Para el mes de tratamiento presentó resolución de las lesiones con mínima pérdida de sustancia (Figuras 2 y 3 ).

\section{DISCUSIÓN}

El objetivo de presentar este caso clínico es describir la presentación de una patología rara, de difícil diagnóstico y que debido a su muy baja prevalencia es poco sospechada. Además, es dar evidencia al beneficio del uso de prostaglandinas E1 ante el alto riesgo de amputación.

La presentación típica involucra a hombres jóvenes (edad promedio 45 años), inferior a la de presentación de arteriopatía aterosclerótica, con historia de 10 años de tabaquismo que refieren claudicación moderada/severa y evolucionan rápidamente a la isquemia crítica de miembros (ICM). Se presenta con episodios de dolor a predominio nocturno, coloración cianótica (signo de dedo azul: SDA) y lesiones de miembros ${ }^{1,3}$. Existe compromiso concomitante de MS, sea clínicamente manifiesto (50\%) o evidenciado mediante test de Allen o arteriografía ${ }^{4}$. El diagnóstico se efectúa por exclusión de otras causas de ICM asociado a un número de catracertísticas propias enumeradas por diversos autores en sistemas de puntuación que permiten realizar el diagnóstico de tromboangeítis obliterante con distintos grados de probabilidad entre los que se destacan los criterios de Papa et al. (Tabla 1) ${ }^{5}$ y los de Olin et al. ${ }^{6}$. No existen tests de laboratorio ni exámenes complementarios específicos. Los pulsos periféricos pueden estar conservados. Entre los exámenes deben solicitarse marcadores de enfermedades hematológicas y autoinmunes ${ }^{1}$. Con respecto a la arteriografía, sus hallazgos pueden ser sugestivos, pero no patognomónicos. Las anormalidades pueden observarse incluso en extremidades que no se hallan aún comprometidas clínicamente por lo que se recomienda arteriografía de cuatro miembros. Se observan arterias totalmente proximales normales con compromiso obstructivo de vasos infrapoplíteos y antebraquiales, particularmente las palmares y digitales. La afectación suele ser segmentaria, con transición abrupta entre un vaso normal y una oclusión. En los casos crónicos, se encuentran abundantes colaterales, en forma de sacacorchos ${ }^{1,3}$. Puede asociarse a tromboflebitis migratoria superficial y menos frecuentemente a compromiso visceral ${ }^{4,7}$. 


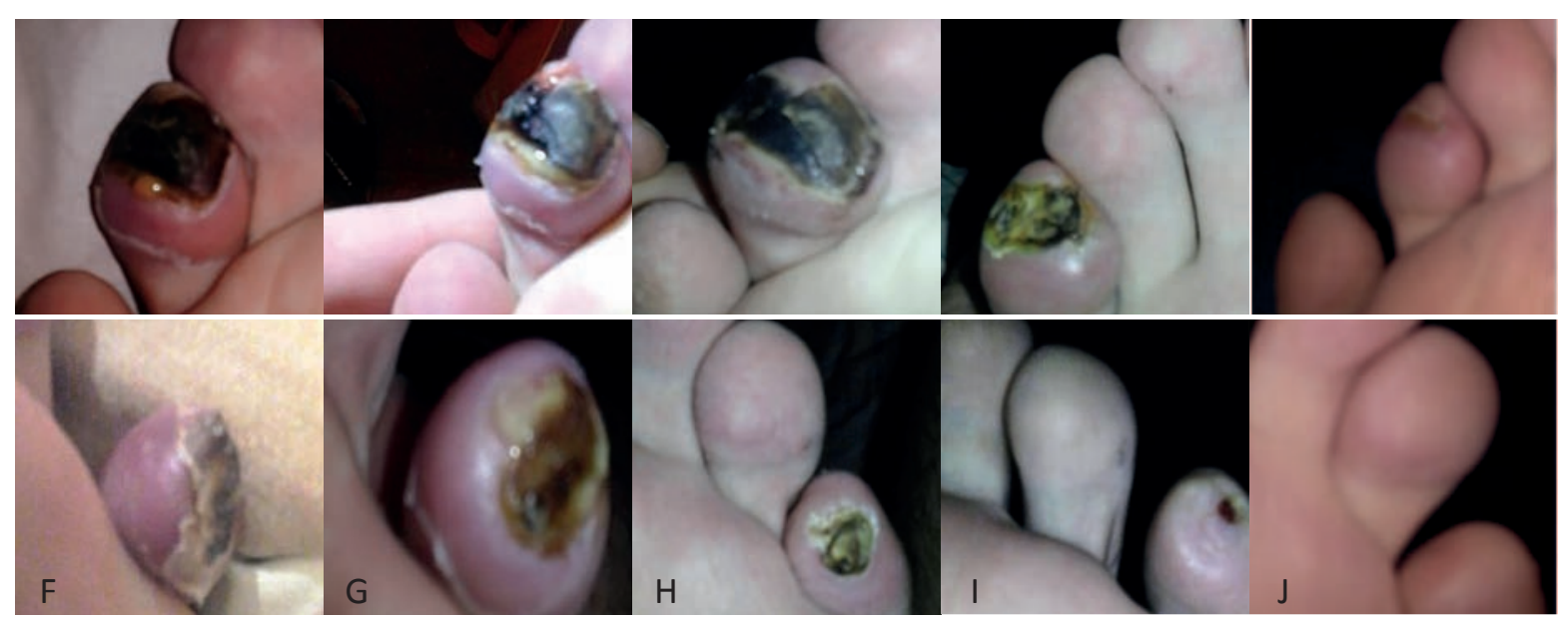

Figura 2. Evolución postratamiento de las lesiones con alprostadil y procaína. F: día 1. H: tratamiento completo. J: 2 meses postratamiento.

El patrón histológico consiste en un trombo inflamatorio con preservación de la lámina elástica interna, a diferencia de lo que sucede en las vasculitis. En la fase crónica, puede que solo se observe obstrucción fibrótica de arterias ${ }^{1}$.

Es importante descartar embolismo de colesterol; ambas patologías presentan dolor y SDA en presencia de pulsos periféricos, sin laboratorio específico. Aunque la biopsia demuestre los cristales de colesterol, la histología no siempre se correlaciona con la clínica. Además, eventos de traumas, procedimientos invasivos, anticoagulantes/trombolíticos pueden desencadenar el cuadro meses después ${ }^{8}$. Otras fuentes embolígenas proximales deben descartarse mediante ecocardiograma y angio-TAC.

La abstinencia completa de tabaco en todas sus formas y vías (mascar, suplementos de nicotina) es la condición sine qua non del tratamiento de la EB aunque no siempre detiene las exacerbaciones; si persiste, lleva a la amputación ${ }^{1,4}$. En este caso, si bien el paciente disminuyó el consumo del tabaco, mantuvo el hábito hasta su internación.

La farmacoterapia (esteroides, calcioantagonistas, pentoxifilina, vasodilatadores, antiplaquetarios, anticoagulantes) es generalmente inefectiva, al igual que los AINE y opiáceos ${ }^{8,10}$. Las medidas locales se reducen a los cuidados habituales de las heridas y al uso anecdótico de cámara hiperbárica.

Por otra parte, los avances técnicos en el intervencionismo vascular tienen escaso impacto ante el compromiso de arterias digitales como en el caso que nos ocupa. En tanto, la infusión intraarterial de factores angiogénicos no constituye aún un tratamiento universalmente aceptado. Es magro también el aporte de la cirugía, que se reduce a resultados controversiales con la simpatectomía y el implante de estimuladores de médula espinal ${ }^{11}$.

En 1990, se reportó la eficacia del iloprost para mejorar los síntomas y acelerar la resolución de lesiones al reducir la pérdida tisular y las amputaciones ${ }^{10}$. Posteriormente, se han reportado resultados favorables en distintas series con el alprostadil ${ }^{8,12}$. Además, como todo tratamiento integral, se deben considerar medidas preventivas para prevenir la injuria mecánica, térmica y química (uso de calzado adecuado, evitar vasoconstrictores, exposición al frío $)^{12}$.

\section{CONCLUSIONES}

Describimos el caso de una patología que, como se desprende del relato, es poco sospechada. Si bien de baja prevalencia, presenta un carácter altamente incapacitante y las demoras en el diagnóstico y tratamiento empeoran el pronóstico. En efecto, el interminable vía crucis de nuestro paciente, más allá de sus padecimientos, lo puso en riesgo de amputación sin que se le indicara en sus inicios, siquiera, la cesación del hábito tabáquico. Creemos, además, que el uso de alprostadil en este caso constituyó un puente para el salvataje de las extremidades hasta la reversión de los efectos residuales del tabaquismo.

\section{BIBLIOGRAFÍA}

1. Goiriz-Valdés R, Fernández-Herrera J. Enfermedad de Buerger (tromboangeítis obliterante). Actas Dermosifiliogr 2005; 96(9):553-62.

2. Papa MZ, Bass A, Adar R, et al. Autoinmune mechanisms in thromboangiitis obliterans (Buerger's disease): The role of tobacco antigen and the major histocompatibility complex. Surgery. 1992;111:527-31.

3. NarváezJ,BianchiMM, SantoP, Castellvil.Síndromedel dedo azul. Seminarios dela Fundación Españolade Reumatología. Semin Fund Esp Reumatol 2011;12(1):2-9.

4. Wysokinski WE, Kwiatkowska W, Sapian-Raczkowska B, et al. Sustained classic clinica spectrum of thromboangiitis obliterans (Buerger'sdisease). Angiology 2000;51(2):141-50.

5. A point scoring system for the clinical diagnosis of Buerger's disease. Eur J VascEndovasc Surg. 1996; 11(3):335-9).

6. Olin JW, Young JR, Graor RA, Ruschhaupt WF, Bartholomew JR. The changing clinical spectrum of thromboangiitis obliterans (Buerger's disease). Circulation 1990;82:Supp/ IV:IV-3-IV-8.

7. Çalgüneri M, ÖztürkA, AyH, et al. Buerger's disease with multisystemic involvement. Case report and review of the literature. Angiology 2004;55:325-7.
8. Vincent Falanga, MD; MichaelJ. Fine, MD; Wishwa N. Kapoor, MD. The Cutaneous Manifestations of Cholesterol Crystal Embolization.Arch Dermatol. 1986;122(10):1194-8

9. Karles-Ernotte AJ, Bermúdez-Posada A, Rincón-Sánchez HM, et al. Efecto terapéutico del alprostadil en pacientes con isquemia crítica terminal de los miembros inferiores. Rev Colomb Cardiol. 2017;24(1):60-65.

10. Fiessinger JN, Schafer M. Trial of iloprost versus aspirin treatment for critical limb ischaemia of thromboangitis obliterans. Lancet 1990;335:555-7.

11. Bozkurt AK, KöksalC, Demirbas MY, et al. A randomized trial of intravenous iloprost (a stable prostacyclin analogue) versus lumbar sympathectomyin the management of Buerger's disease. Int Angiol 2006;25:162-7.

12. Mangiafico RA, Ortiz Limonta D y Ortiz Guilarte A. Preservation of extremities in patients with occlussive peripheral artery disease. MEDISAN 2016; 20(2):227-33.

13. Mangiafico M. Medical treatment of critical limb ischemia: current stateand future directions. Current vascular pharmacology. 2011;9:658-76. 